\title{
LOCAL SOLVABILITY OF LEFT INVARIANT DIFFERENTIAL OPERATORS ON THE HEISENBERG GROUP
}

\author{
LINDA PREISS ROTHSCHILD ${ }^{1}$
}

\begin{abstract}
Representation-theoretic criteria are given for the local solvability of left invariant differential operators homogeneous under dilations.
\end{abstract}

In this note we prove that a sufficient condition for the local solvability of a homogeneous left invariant differential operator on the Heisenberg group is that the image of the operator under any infinite dimensional irreducible unitary representation be invertible. This result was conjectured by Rockland [7], and the proof given here is based mainly on his approach.

Let $H^{n}$ be the Heisenberg group of dimension $2 n+1$; i.e. $H^{n}$ is the simply connected nilpotent Lie group whose Lie algebra $\mathfrak{h}^{n}$ is spanned by $X_{1}, X_{2}, \ldots, X_{n}, Y_{1}, Y_{2}, \ldots, Y_{n}, T$, satisfying $\left[X_{j}, Y_{k}\right]=\delta_{j k}$, with all other commutators zero. A differential operator $D$ on $H^{n}$ is left invariant if $D\left({ }^{8} f\right)={ }^{g}(D f)$, for any $g \in H^{n}$ and any function $f$; here ${ }^{8} f$ is the function $x \rightarrow f(g x)$. By the exponential map any $Z \in \mathfrak{h}^{n}$ may be identitifed with a left invariant differential operator:

$$
(Z f)(g)=\left[\frac{d}{d t} f(g \exp t Z)\right]_{t=0} .
$$

Conversely, any left invariant differential operator on $H^{n}$ may be written as a polynomial in the elements of $\mathfrak{h}^{n}$ with constant coefficients.

The algebra $\mathfrak{h}^{n}$ carries a family of automorphisms $\delta_{r}$, defined for $r>0$ by

$$
\delta_{r}\left(X_{j}\right)=r X_{j}, \quad \delta_{r}\left(Y_{j}\right)=r Y_{j}, \quad j=1,2, \ldots, n \text {, and } \delta_{r}(T)=r^{2} T .
$$

$\delta_{r}$ may be extended to polynomials on $\mathfrak{h}^{n}$ by defining

$$
\delta_{r}\left(Z_{1} Z_{2} \cdots Z_{k}\right)=\delta_{r}\left(Z_{1}\right) \delta_{r}\left(Z_{2}\right) \cdots \delta_{r}\left(Z_{k}\right) .
$$

$\delta_{r}$ is well defined by the Birkhoff-Poincaré-Witt theorem (e.g. [2]). A left invariant differential operator is homogeneous of degree $d$ if $\delta_{r}(D)=r^{d} D$.

Every irreducible unitary representation $\pi$ of $H^{n}$ on a Hilbert space $V$ determines an irreducible representation of $\mathfrak{h}^{n}$, also denoted $\pi$, by

$$
\pi(Z) v=\left[\frac{d}{d t} \pi(\exp t Z) v\right]_{t=0}, \quad Z \in \mathfrak{h}^{n}, v \in V
$$

Received by the editors March 23, 1978.

AMS (MOS) subject classifications (1970). Primary 58G15, 22E30; Secondary 35H05, $22 \mathrm{E} 45$.

Key words and phrases. Heisenberg group, local solvability, left invariant operator.

${ }^{1}$ Partially supported by NSF grant MCS 77-01155, and an A.P. Sloan Fellowship. 
The irreducible unitary representation of $H^{n}$ are of two types (e.g. [5]):

(i) Finite-dimensional: $V=\mathrm{C}$, and the representations are parametrized by $(a, b) \in R^{2 n}$ with

$$
\pi_{(a, b)}\left(X_{j}\right)=i a_{j}, \quad \pi_{(a, b)}\left(Y_{j}\right)=i b_{j}, \text { and } \pi(T)=0 .
$$

(ii) Infinite-dimensional: $V=L^{2}\left(\mathbf{R}^{n}\right)$, and the representations are parametrized by $\lambda \in \mathbf{R}-\{0\}$ with

$$
\begin{aligned}
\pi_{\lambda}\left(X_{j}\right)=|\lambda|^{1 / 2} \frac{\partial}{\partial x_{j}}, \pi_{\lambda}\left(Y_{j}\right) & =i|\lambda|^{1 / 2}(\operatorname{sgn} \lambda) x_{j}, \\
j & =1,2, \ldots, n \text { and } \pi_{\lambda}(T)=i \lambda .
\end{aligned}
$$

A differential operator $D$ is locally solvable at a point $x_{0}$ if there is a neighborhood $U$ of $x_{0}$ such that $D C^{\infty}(U) \subset C_{0}^{\infty}(U)$, where $C^{\infty}$ and $C_{0}^{\infty}$ denote smooth functions and smooth functions with compact support, respectively. For left invariant differential operators it suffices to consider $x_{0}=0$, the identity. The reader is referred to the survey article by Helgason [3] on local solvability for general Lie groups and to [6] or [8] for local solvability of bi-invariant differential operators.

The result may now be stated precisely.

THEOREM. Let $P$ be a homogeneous left invariant differential operator on the Heisenberg group $H^{n}$. Then $P$ is locally solvable if $\pi(P)$ has a bounded left inverse for every infinite dimensional irreducible unitary representation $\pi$ of $\boldsymbol{H}^{n}$.

Lemma 1. It suffices to prove that there exists $N>0$ such that for every $v \in C_{0}^{\infty}\left(H^{n}\right)$

$$
P u=T^{N} v
$$

has a solution $u \in C^{\infty}\left(H^{n}\right)$.

Proof. Since $T=\partial / \partial t$ in suitable coordinates, one may obtain $v_{1} \in C^{\infty}$ satisfying

$$
T^{N} v_{1}=f
$$

by integrating $f N$ times in $t$. Now if $U \subset H^{n}$ has compact closure, choose $v \in C_{0}^{\infty}$ such that $v \equiv v_{1}$ on $U$. Then if $u \in C^{\infty}$ satisfies (2),

$$
P u=T^{N} v_{1}=f \text { in } U .
$$

We now prove the theorem by constructing a solution of (2), using the Plancherel Theorem for $H^{n}$ (e.g. [5]). If $\varphi \in C_{0}^{\infty}$, and $\pi_{\lambda}$ is an infinite dimensional irreducible unitary representation of $H^{n}$, then the operator

$$
\pi_{\lambda}(\varphi)=\int_{H^{n}} \varphi(x) \pi_{\lambda}(x) d x
$$

is of trace class. In particular, if $\left\{e_{i}\right\}$ is any orthonormal basis of $L^{2}\left(\mathbf{R}^{n}\right)$, then

$$
\operatorname{tr}\left(\pi_{\lambda}(\varphi)\right)=\sum_{i}\left(\pi_{\lambda}(\varphi) e_{i}, e_{i}\right)<\infty
$$


The Plancherel Theorem for $H^{n}$ states that for $\varphi \in C_{0}^{\infty}\left(H^{n}\right)$,

$$
\|\varphi\|_{L^{2}}=\int_{\mathbf{R}-\{0\}} \operatorname{tr}\left(\pi_{\lambda}(\varphi) \pi_{\lambda}(\varphi)^{*}\right)|\lambda|^{n} d \lambda
$$

where $\pi_{\lambda}(\varphi)^{*}$ is the formal adjoint, and

$$
\varphi(0)=\int_{\mathbf{R}-\{0\}} \operatorname{tr}\left(\pi_{\lambda}(\varphi)\right)|\lambda|^{n} d \lambda .
$$

Now suppose $P$ is homogeneous of degree $\alpha>0$. Then

$$
\pi_{\lambda}(P)=\left\{\begin{array}{lc}
|\lambda|^{\alpha / 2} \pi_{1}(P), & \lambda>0, \\
|\lambda|^{\alpha / 2} \pi_{-1}(P), & \lambda<0,
\end{array}\right.
$$

by (1).

The hypothesis of the theorem is then that $\pi_{1}(P)$ and $\pi_{-1}(P)$ have bounded inverses, to be denoted $B_{1}$ and $B_{-1}$. Following [7], we first reduce to the case where $B_{1}$ and $B_{-1}$ are of trace class. This is accomplished by replacing $P$ by $P Q$, where

$$
Q=\left(\sum X_{j}^{2}+Y_{j}^{2}\right)^{N^{\prime}}
$$

for large $N^{\prime}$. Indeed, it is shown in [7, Lemma 3.3] that $\pi_{1}(Q)^{-1}$ is of trace class. Since the product of a bounded operator and an operator of trace class is again of trace class, the inverse of $\pi_{1}(P Q)=\pi_{1}(P) \pi_{1}(Q)$ is of trace class. $P Q$ again satisfies the hypotheses of the theorem and the local solvability of $P Q$ implies that of $P$. Replacing $P$ by $P Q$ if necessary, we may assume that $B_{1}$ and $B_{-1}$ are of trace class.

To solve (2), let $u$ be the linear mapping $C_{0}^{\infty}\left(H^{n}\right) \rightarrow \mathrm{C}$ defined by

$$
\varphi \rightarrow u(\varphi)=\int(i \lambda)^{N} \operatorname{tr}\left(\pi_{\lambda}(\tilde{v}) \pi_{\lambda}(\varphi)\left(\pi_{\lambda}(P)\right)^{-1}\right)|\lambda|^{n} d \lambda
$$

for some large $N$, where $\tilde{v}(x)=v\left(x^{-1}\right)$. We shall first show that $u$ is an $L^{2}$ function by proving

$$
|u(\varphi)| \leqslant C\|\varphi\|_{L^{2}}
$$

By (6), the absolute value of the right-hand side of (7) is bounded by

$$
\begin{aligned}
\left.\left|\int_{\lambda>0}\right| \lambda\right|^{N-\alpha / 2}\left|\left(\operatorname{tr}\left(\pi_{\lambda}(\tilde{v}) \pi_{\lambda}(\varphi) B_{1}\right)\right)\right||\lambda|^{n} d \lambda \mid \\
+\left.\left.\left|\int_{\lambda<0}\right| \lambda\right|^{N-\alpha / 2}\left|\operatorname{tr}\left(\pi_{\lambda}(\tilde{v}) \pi_{\lambda}(\varphi) B_{-1}\right)\right| \lambda\right|^{n}|d \lambda| .
\end{aligned}
$$


If $\alpha>0$ is arbitrary, then

$$
\begin{aligned}
\left.\left|\int_{\lambda>0}\right| \lambda\right|^{N-\alpha / 2}\left|\left(\operatorname{tr}\left(\pi_{\lambda}(\tilde{v}) \pi_{\lambda}(\varphi) B_{1}\right)\right)\right||\lambda|^{n} d \lambda \mid \\
\leqslant\left.\left|\int_{0<\lambda<\alpha}\right| \lambda\right|^{N-\alpha / 2}\left|\left(\operatorname{tr}\left(\pi_{\lambda}(\tilde{v}) \pi_{\lambda}(\varphi) B_{1}\right)\right)\right||\lambda|^{n} d \lambda \mid \\
\quad+\left.\left|\int_{\alpha<\lambda<\infty}\right| \lambda\right|^{N-\alpha / 2}\left|\left(\operatorname{tr}\left(\pi_{\lambda}(\tilde{v}) \pi_{\lambda}(\varphi) B_{1}\right)\right)\right||\lambda|^{n} d \lambda \mid .
\end{aligned}
$$

The generalized Schwartz inequality for arbitrary operators $C, D$ of trace class states that

$$
|\operatorname{tr}(C D)| \leqslant\left(\operatorname{tr}\left(C C^{*}\right)\right)^{1 / 2}\left(\operatorname{tr}\left(D D^{*}\right)\right)^{1 / 2} .
$$

The first integral on the right of $(10)$ is bounded by

$$
\left[\int_{0}^{a}|\lambda|^{2(N-\alpha / 2)+n} d \lambda\right]^{1 / 2}\left[\int_{0}^{\lambda}\left|\operatorname{tr}\left(\pi_{\lambda}(\tilde{v}) \pi_{\lambda}(\varphi) B_{1}\right)\right|^{2}|\lambda|^{n} d \lambda\right]^{1 / 2}
$$

by the standard Schwartz inequality. If $2(N-\alpha / 2)+n>-1$ i.e. if

$$
N>(-(n+1)+\alpha) / 2
$$

then the first factor in (12) is finite, say bounded by $C>0$. By (11), (12) is bounded by

$$
C\left[\operatorname{tr}\left(B_{1} B_{1}^{*}\right)\right]^{1 / 2}\left[\int_{0}^{\lambda} \operatorname{tr}\left(\pi_{\lambda}(\tilde{v}) \pi_{\lambda}(\varphi) \pi_{\lambda}(\varphi)^{*} \pi_{\lambda}(\tilde{v})^{*}\right)|\lambda|^{n} d \lambda\right]^{1 / 2} .
$$

Now recall that the group convolution of, say, two functions $\psi, \chi \in C_{0}^{\infty}$ is defined as

$$
(\psi * \chi)(g)=\int \psi\left(x^{-1}\right) \chi(x g) d x .
$$

It is well known, and easy to check, that

$$
\pi_{\lambda}(\psi * \chi)=\pi_{\lambda}(\psi) \pi_{\lambda}(\chi)
$$

Hence (13) is bounded by

$$
\left.\left.C^{\prime}\left|\int_{0}^{\lambda} \operatorname{tr}\left(\pi_{\lambda}(\tilde{v} * \varphi) \pi_{\lambda}(\tilde{v} * \varphi)^{*}\right)\right| \lambda\right|^{n} d \lambda\right|^{1 / 2}=C^{\prime}\|\tilde{v} * \varphi\|_{L^{2}}
$$

by (4). By the general Hausdorff-Young inequality

$$
\|\tilde{v} * \varphi\|_{L^{2}} \leqslant\|\tilde{v}\|_{L^{1}}\|\varphi\|_{L^{2}} \leqslant C^{\prime}\|\varphi\|_{L^{2}}
$$

Estimation of the second integral on the right-hand side of (10) is similar except that (12) is replaced by

$$
\left[\int_{a}^{\infty}|\lambda|^{2(N-\alpha / 2)+n-N^{\prime}} d \lambda\right]^{1 / 2}\left[\int_{0}^{\lambda}\left|\operatorname{tr}\left(\lambda^{N^{\prime}} \pi_{\lambda}(\tilde{v}) \pi_{\lambda}(\varphi) B_{1}\right)\right||\lambda|^{n} d \lambda\right]^{1 / 2} .
$$

Now $(i \lambda)^{N^{\prime}}=\pi_{\lambda}\left(T^{N^{\prime}}\right)$ and $\pi_{\lambda}\left(T^{N^{\prime}}\right) \pi_{\lambda}(\tilde{v})=\pi_{\lambda}\left(\widetilde{T^{N^{\prime}}} v\right)$. If $N^{\prime}$ is chosen so large 
that the first factor is finite, (16) is bounded by

$$
\begin{aligned}
& C^{\prime \prime}\left\{\int_{0}^{\lambda} \operatorname{tr}\left(\pi_{\lambda}\left(\widetilde{T^{N^{\prime}} v}\right) \pi_{\lambda}(\varphi) \pi_{\lambda}(\varphi)^{*} \pi_{\lambda}\left(\widetilde{T^{N^{\prime}} v}\right)^{*}\right)|\lambda|^{n} d \lambda\right\}^{1 / 2}
\end{aligned}
$$

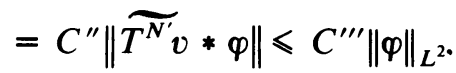

Combining these estimates and similar ones for the other integral in (9) gives (8). This argument is very similar to that given in [7].

To show that the $L^{2}$ function $u$ is actually smooth it suffices, by Sobolev's Lemma, to show that all distributional derivatives of $u$ are in $L^{2}$. We shall show that if $D$ is any left invariant differential operator on $H^{n}$, then

$$
|u(D \varphi)| \leqslant C_{D}\|\varphi\|_{L^{2}}, \quad \varphi \in C_{0}^{\infty}\left(H^{n}\right) .
$$

In proving (17) it will be convenient to use right invariant differential operators. If $Z \in \mathfrak{h}^{n}$, then there is a right invariant vector field $Z^{r}$ determined by $Z$, i.e.

$$
Z^{r} f(g)=\left[\frac{d}{d t} f(\exp t Z \cdot g)\right]_{t=0}
$$

If $D=Z_{1} Z_{2} \cdots Z_{k}$ is a left invariant differential operator with $Z_{j} \in \mathfrak{h}^{n}$, $1 \leqslant j \leqslant k$, denote by $D^{r}$ the right invariant vector field $D^{r}=$ $Z_{k}^{r} Z_{k-1}^{r} \cdots Z_{1}^{r}$.

LEMMA 2. $D \psi * \chi=\psi * D^{r} \chi$ for any left invariant differential operator $D$ and any $\psi, \chi \in C_{0}^{\infty}\left(H^{n}\right)$.

Proof. It suffices to assume $D=Z \in \mathfrak{h}^{n}$. Then

$$
\begin{aligned}
Z \psi * \chi & =\int\left[\frac{d}{d t}\left(\psi\left(g x^{-1} \exp t Z\right) \chi(x)\right)\right]_{t=0} d x \\
& =\int\left[\frac{d}{d t} \psi\left(g x^{-1}\right) \chi((\exp t Z) x)\right]_{t=0} d x=\psi * Z^{r} \chi
\end{aligned}
$$

(The second equality is obtained by making the change of variables $x \rightarrow$ ( $\exp t Z) x$ and using the fact that the measure $d x$ is left invariant.)

To prove the inequality (17), one proceeds as above, but with $\varphi$ replaced by $D \varphi$. Since

$$
\begin{gathered}
\operatorname{tr}\left(\pi_{\lambda}(\tilde{v}) \pi_{\lambda}(D \varphi) \pi_{\lambda}(D \varphi)^{*} \pi_{\lambda}(\tilde{v})^{*}\right)=\operatorname{tr}\left(\pi_{\lambda}(D \varphi)^{*} \pi_{\lambda}(\tilde{v}) \pi_{\lambda}(\tilde{v})^{*} \pi_{\lambda}(D \varphi)\right) \\
\int_{0<\lambda<a}|\lambda|^{N} \operatorname{tr}\left(\pi_{\lambda}(\tilde{v}) \pi_{\lambda}(D \varphi)\left(\pi_{\lambda}(P)\right)^{-1}\right)|\lambda|^{n} d \lambda \\
\leqslant C\|D \varphi * \tilde{v}\|_{L^{2}}=C\left\|\varphi * D^{r} \tilde{v}\right\|_{L^{2}} \leqslant C^{\prime}\|\varphi\|_{L^{2}}
\end{gathered}
$$

Similarly,

$$
\begin{aligned}
& \int_{0<\lambda<\infty}|\lambda|^{N} \operatorname{tr}\left(\pi_{\lambda}(\tilde{v}) \pi_{\lambda}(D \varphi)\left(\pi_{\lambda}(P)\right)^{-1}\right)|\lambda|^{n} d \lambda \\
& \leqslant C\left\|D \varphi * T^{N^{\prime}} \tilde{v}\right\|_{L^{2}}=C\left\|\varphi * D^{r} T^{N^{\prime}} \tilde{v}\right\|_{L^{2}} \leqslant C^{\prime}\|\varphi\|_{L^{2 *}}
\end{aligned}
$$


The estimates for $\lambda<0$ are the same. This completes the proof that $u$ is a $C^{\infty}$ function. Finally, it is a simple formal calculation to show that $u$ satisfies (2) i.e. that $u\left(P^{\tau} \varphi\right)=T^{N} v(\varphi)$ for all $\varphi \in C_{0}^{\infty}\left(H^{n}\right)$, where $P^{\tau}$ is the formal transpose of $P$. Indeed, since $\pi_{\lambda}\left(P^{\tau} \varphi\right)=\pi_{\lambda}(\varphi) \pi_{\lambda}(P)$, and $(i \lambda)^{N} \pi_{\lambda}(\tilde{v})=$ $\pi_{\lambda}(\tilde{v}) \pi_{\lambda}\left(T^{N}\right)=\pi_{\lambda}\left(\widetilde{T^{N} v}\right)$

$$
\begin{aligned}
u\left(P^{\tau} \varphi\right) & =\int(i \lambda)^{N} \operatorname{tr}\left(\pi_{\lambda}(v) \pi_{\lambda}\left(P^{\tau} \varphi\right)\left(\pi_{\lambda}(P)\right)^{-1}\right)|\lambda|^{N} d \lambda \\
& =\int \operatorname{tr}\left(\pi_{\lambda}\left(\widetilde{T^{N} v}\right) \pi_{\lambda}(\varphi)\right)|\lambda|^{N} d \lambda \\
& =\int \operatorname{tr}\left(\widetilde{T^{N} v} * \varphi\right)|\lambda|^{N} d \lambda \\
& =\left(\widetilde{T^{N} v} * \varphi\right)(0), \quad \text { by the Plancherel theorem (5), } \\
& =T^{N} v(\varphi), \quad \text { by (14). }
\end{aligned}
$$

This completes the proof of the theorem.

REMARKS. 1. Rockland [7] proves that if $\pi(P)$ and $\pi\left(P^{*}\right)$ have trivial $L^{2}$ kernels for all irreducible unitary representations, then $P$ is hypoelliptic and locally solvable. Using pseudodifferential operators, Miller [4] was able to prove this theorem assuming the hypothesis only for $\pi(P)$ has no rapidly decreasing eigenfunction of eigenvalue zero, for all $\pi$. The possible relationship between hypoellipticity and invertibility of representations on $H^{n}$ was first observed by Folland and Stein [1]. (See also [9].)

2. The author has proved local solvability for some classes of homogeneous left invariant differential operators on more general nilpotent Lie groups. The proofs involve more detailed harmonic analysis [10].

ADDED IN PROOF. These results have been proved independently by $G$. Lion.

\section{REFERENCES}

1. G. Folland and E. M. Stein, Estimates for the $\bar{\partial}_{b}$-complex and analysis on the Heisenberg group, Comm. Pure Appl. Math. 27 (1974), 429-522.

2. S. Helgason, Differential geometry and symmetric spaces, Academic Press, New York, 1962.

3. __ Solvability of invariant differential operators on homogeneous manifolds, CIME Seminar (1975).

4. K. G. Miller, Hypoellipticity on the Heisenberg group (preprint).

5. L. Pukanszky, Lecons sur les représentations des groupes, Dunod, Paris, 1967.

6. M. Raï, Solutions élémentaires des opérateurs différentiels bi-invariants sur un group de Lie nilpotent, C. R. Acad. Sci. Paris Sér. A-B 273 (1971), 495-498.

7. C. Rockland, Hypoellipticity on the Heisenberg group-representation-theoretic criteria, Trans. Amer. Math. Soc. 240 (1978), 1-52.

8. F. Rouvière, Sur la résolubilité locale des opérateurs bi-invariants, Ann. Scuola Norm. Sup. Pisa Cl. Sci. (2) 3 (1976), 231-244.

9. L. P. Rothschild and E. M. Stein, Hypoelliptic differential operators and nilpotent groups, Acta Math. 137 (1976), 247-320.

10. L. P. Rothschild, Local solvability of second order differential operators (preprint).

Department of Mathematics, University of Wisconsin, Madison, Wisconsin 53706 